\title{
Review of: PLoS Biology-A Freely Available, Open Access Online Journal
}

\section{Todd Eckdahl*}

\author{
Department of Biology, Missouri Western State College, 4525 Downs Drive, Saint Joseph, MO 64507
}

\section{PLOS BIOLOGY AS A BOLD EXPERIMENT}

The Public Library of Science (PLoS) (http://www.plos.org/) is a nonprofit organization of scientists and physicians that is conducting a high profile and ambitious experiment. It is testing the refreshing idea that scientific knowledge can be made freely available throughout the world to scientists, educators, students, physicians, patients, policy makers and voting citizens. PLoS is committed to the idea that scientific research should be published in a timely manner in an open-access environment with no restrictions on use.

The probability of success of the PLoS endeavor is increased because of its leadership and its growing widespread support. Cofounders Harold Varmus, Pat Brown, and Michael Eisen (Figure 1) began their efforts in October 2000 by circulating an open letter of support (http://www.plos.org/support/ openletter.shtml) petitioning publishers to make research reports available free of charge in online libraries such as PubMed Central (http://www.pubmedcentral.nih.gov/). Although signed by more than 30,000 scientists from around the world, the letter failed to have the desired impact. The bold decision was then made for PLoS to establish its own models for freely available, open-access online journals. A grant of $\$ 9$ million from the Gordon and Betty Moore Foundation (http:// www.moore.org/) allowed the formation of the PLoS editorial board and staff. The organization made plans to develop a premier Biology journal first, followed by one devoted to Medicine. Future PLoS journals include Chemistry, Computer Science, Genetics, Oncology, and a host of other titles. The first issue of PLoS Biology (http://www.plosbiology.org) was published in October 2003, and a carefully designed experiment began to test an idea that would change the way we access scientific research (Figure 2).

The success of thePLoS Biologyexperiment depends on the exploration of business practices that make sense to publishers. The business model that has been adopted for PLoS Biologyis one that its editors hope will become popular with other journals. The idea is for authors, in partnership with their institutions and funding agencies, to bear the cost of publishing their work so that online access can be given free of charge to the public. A publication fee of $\$ 1500$ is charged, payable by the author, institution, or funding agency. However, the ability to pay the fee will not be used in decisions about publication.

DOI: $10.1187 /$ cbe.04-01-0026

*Corresponding author. E-mail address: eckdahl@mwsc.edu.

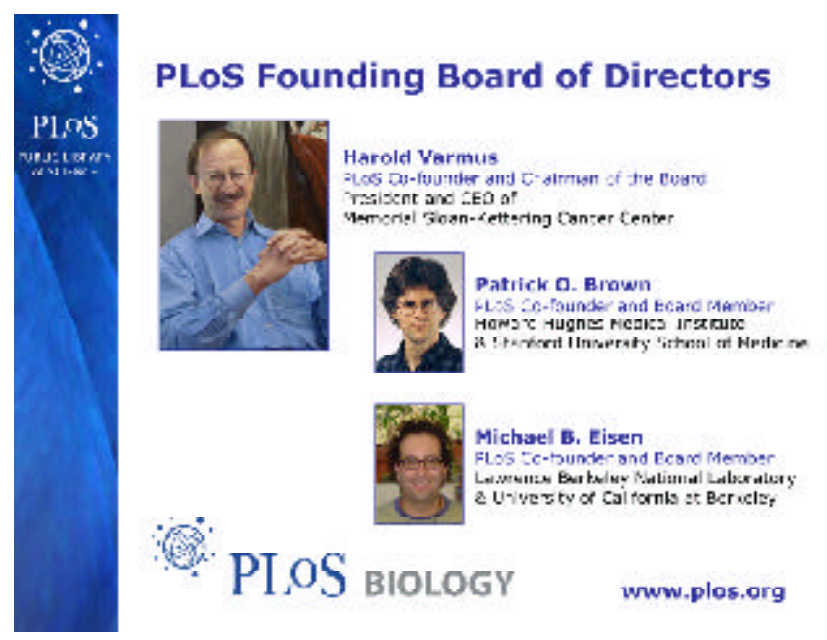

Figure 1. From PLoS Website (http://www.plos.org/downloads/ PLoSFoundingBoard.jpg) accessed January 14, 2004.

PLoS Biology has established editorial procedures that will ensure timely peer review and publication of articles that are likely to make significant contributions to diverse life science fields. Authors are encouraged to make presubmission inquiries and can expect a response within a few days. Once a manuscript is received, it is routed to reviewers by an editorial board composed of professional editors and academic researchers. The fact that the board includes notable scientists from major research universities, industry, and private research institutes around the world will ensure the publication of high-quality research. Reviewers are requested to complete their evaluations within 7 days. Considering this and the immediacy of online publishing, it is hard to imagine a faster means of publication.

\section{PLoS BIOLOGY AS A FULL-SERVICE JOURNAL}

The founders of PLoS Biology have set their sights high in terms of the quality of the research they intend to publish. Their goal is to publish high-profile original research of great significance in all fields of Biology and in crossover areas with other disciplines. Success in meeting this goal is apparent already. One illustration of this is the presentation by Stein et al. of the genome of a second Caenorhabditis species, a discovery that will have a dramatic impact on the utility of both species for basic research. Another is the report by Starck and Brennecke et al. on the development of a new screen for microRNA targets in 


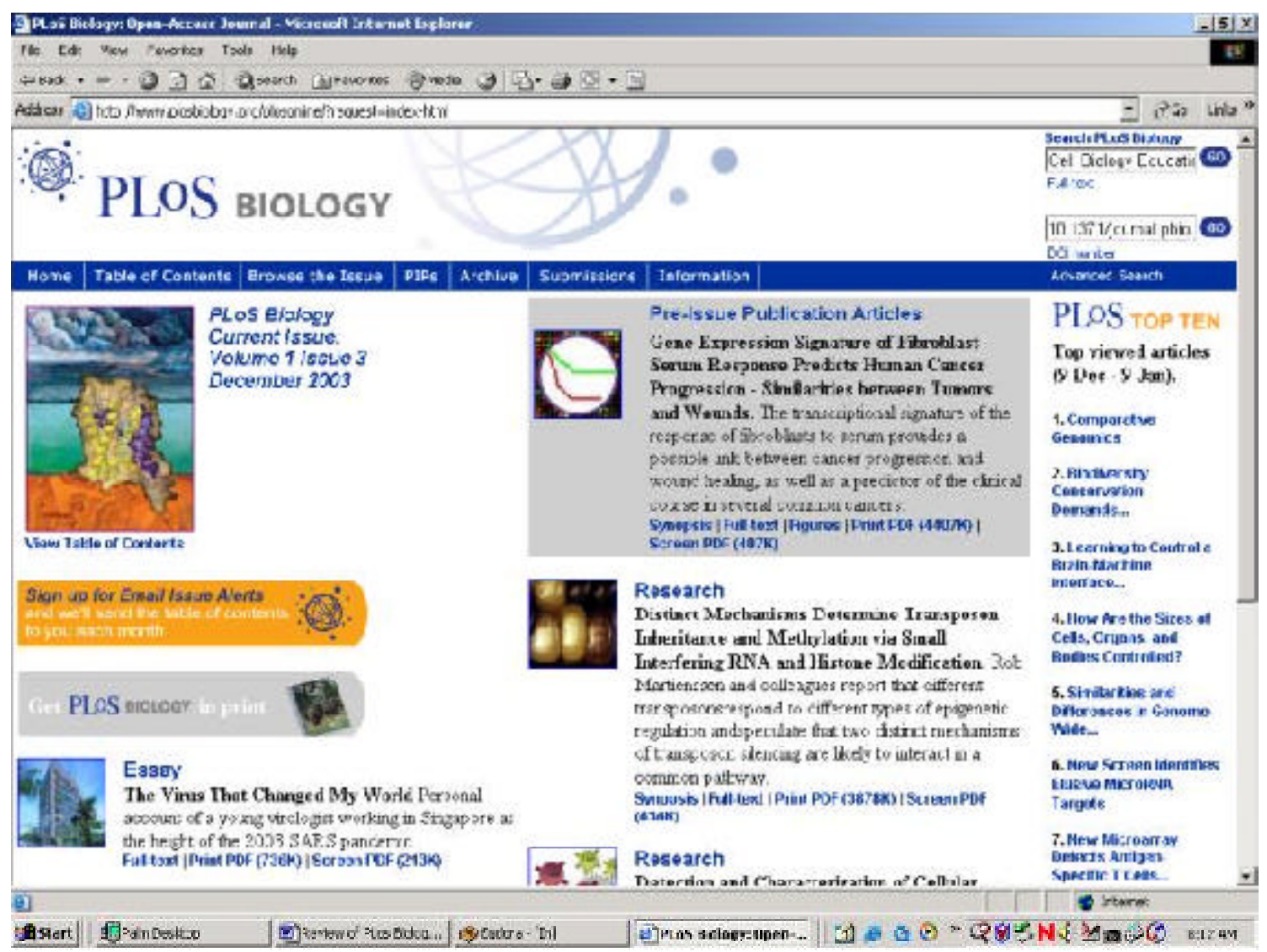

Figure2. PLoS Biology homepage (http://www.plosbiology.org) accessed January 14, 2004.

Drosophila (Figure 3). In yet another example, Fernando et al. describe their use of DNA analysis to show that elephants in Borneo are genetically distinct from other Asian elephants, and therefore worthy of conservation. These and 27 other research articles in the first three monthly issues demonstrate the ability of PLoS Biology to publish world-class primary research. The scope of the research published is broad, from molecules to cells, from organisms to ecosystems. The support of researchers who are leaders in their fields is likely to grow, making PLoS Biology a prestigious publication forum. But PLoS Biology is more than a vehicle for the publication of groundbreaking primary research articles. It is a full-service journal that will appeal to a wide spectrum of readers.

PLoS Biology features a diversity of articles, contributed by research scientists, editors, graduate students, and professional science reporters. For example, the "Editorials" section has been used to this point to discuss the launching of PLoS journals, to describe the way digital object identifiers give persistence to online data, and to champion open-access experiments at PLoS and elsewhere. The journal has also included two "Features" per issue, in which authors present scientific research that is of broad interest to society. A diversity of topics has been addressed here already, from GMO corn to the ecology of infectious disease to the human epigenome project. In addition, a section called "Essays" has allowed authors to present their viewpoints on the SARS epidemic, the impact of genomics on malaria research, and the importance of data sharing in neuroscience.

Another unique feature of PLoS Biology is called "Journal Club." It is an opportunity for postdoctoral scientists and graduate students to describe their personal journey into a field of biological research. It is likely that the Journal Club will convey a sense of enthusiasm about science that will have a positive impact on many young readers. "Unsolved Mysteries" in PLoS Biology highlight interesting but ill-understood aspects of biology that have not yet been the subjects of intense research. Examples include control of the size of cells, organs, and bodies and the control of human skin color variation. The material presented here could serve as a basis for interesting classroom discussions.

Its publishers have also set the stage for PLoSBiology to serve the scientific community. An example of this is a fitting tribute to the neuroscience research career of Patricia Goldman-Rakic, published as an obituary. Another example is the "Community Page," which provides an environment for the presentation of work by organizations and societies who share the PLoS view about free and open access to scientific knowledge. Already, this section has brought attention to the need for legal support to the open communication and use of scientific research, to the role that open access plays in species conservation, and to the importance of political advocacy for basic research.

Clearly, the publishers of PLoS Biology view open access to 


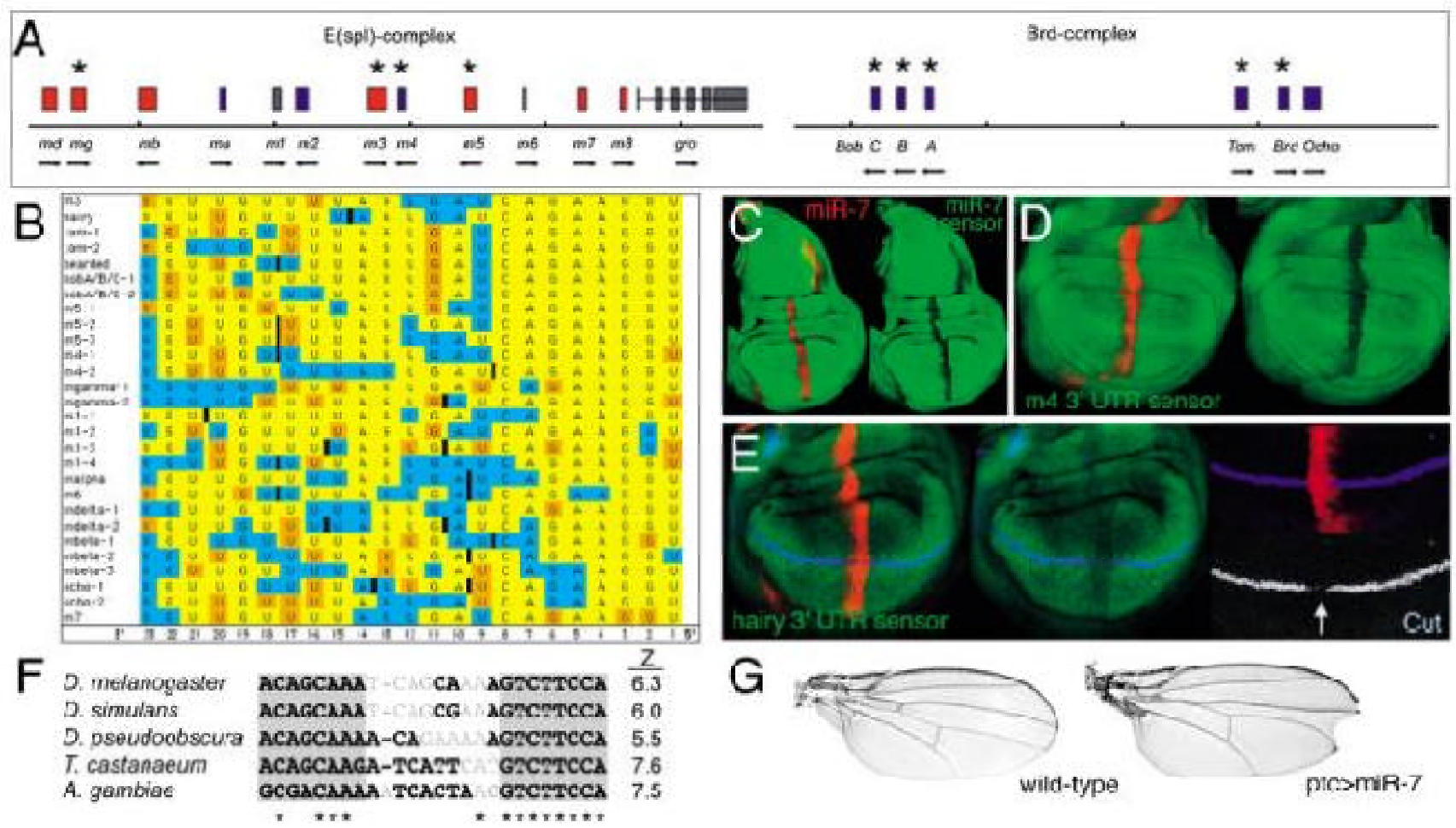

Figure3. Experimental Validation of microRNA Targets, Figure 3 (10.1371_journal.pbio.0000060.g003-L.jpg) from “Identification of Drosophila MicroRNA Targets," by Alexander Stark et al., PLoS Biology volume 1, issue 3.

their journal as more than making scientific research freely available; their intent is to make it accessible to an audience of individuals with diverse backgrounds. Each research article is accompanied by a summary of its purpose, conclusions, and significance for a general audience. These "Synopses" are prepared by science writers and are an immediate and convenient secondary literature connection to primary literature in the same issue. This unique feature of the journal is likely to be useful in a variety of educational settings. High school and undergraduate nonmajors' courses could use the Synopses as a timely link to current research, while lower and upper division life science majors could use them as a bridge to the original research articles. Accessibility to PLoS Biology is also enhanced by "Primers," written by research scientists as introductions to fields of biological research. They include instructional figures, comprehensive reference lists and are written in a succinct textbook style that will be appreciated by students and the lay public. Among the topics of Primers so far are the evolution of the adaptive immune response, microarray analysis, movement control, and the acquisition and distribution of metabolic iron.

\section{OPEN ACCESS PUBLISHING AND SCIENCE EDUCATION}

Open access to current published research free of charge is particularly important in the context of undergraduate education. Especially at undergraduate institutions, access to primary literature continues to be limited because of the financial burden of subscribing to an ever-growing list of journals. This is increasingly frustrating for undergraduate educators who are mindful of the need to engage their students in the process of science at the level of published research. Journals with instant, free, and open access such as PLoS Biology, Cell Biology Education, and over 100 hundred journals at BioMed Central (http:// www.biomedcentral.com/) offer resources now that can enhance science education. What the future holds in this regard, however, depends on whether notice will be taken by the publishing world of the success of these experiments.

What a bright future it would be. Broad and open access to all the scientific literature would completely change the way science education happens. Faculty teaching introductory courses could incorporate examples from the most current literature, conveying to their students a sense of the exciting pace of biological discovery and illustrating its increasing social relevance. Students majoring in a biological discipline could be shown that they are only keystrokes away from the latest research reports. This would serve to strengthen the connection between learning basic concepts and applying them in the course of scientific investigation. And students would be able to learn to take advantage of an online network of published works, databases, and analytical tools. Free and open access to scientific literature would certainly improve the education of tomorrow's teachers, scientists, physicians, and science literate citizens. These dramatic improvements to science education and to the dissemination of science to society depend on the realization of the vision of the Public Library of Science, as illustrated by PLoSBiology. 